\title{
Editorial
}

\section{Advances in Methods for Networked and Cyber-Physical System}

\author{
Jason Gu, ${ }^{1}$ Xiaomei Qi, ${ }^{2}$ Ying Wang, ${ }^{3}$ Fei Liu, ${ }^{4}$ and Chengjin $Z_{\text {hang }}{ }^{5}$ \\ ${ }^{1}$ Department of Electrical and Computer Engineering, Dalhousie University, Halifax, NS, Canada B3J 2X4 \\ ${ }^{2}$ College of Electrical and Electronic Engineering, Shandong University of Technology, Zibo 255049, China \\ ${ }^{3}$ Department of Electrical and Mechatronics Engineering, Southern Polytechnic State University, \\ 1100 South Marietta Parkway Marietta, Marietta, GA 30060, USA \\ ${ }^{4}$ Computer Science and Computer Engineering, La Trobe University, Melbourne, VIC 3086, Australia \\ ${ }^{5}$ School of Mechanical, Electrical \& Information Engineering, Shandong University, Weihai West Road 180, Weihai 264209, China
}

Correspondence should be addressed to Jason $\mathrm{Gu}$; jason.gu@dal.ca

Received 14 April 2014; Accepted 14 April 2014; Published 29 May 2014

Copyright (C) 2014 Jason Gu et al. This is an open access article distributed under the Creative Commons Attribution License, which permits unrestricted use, distribution, and reproduction in any medium, provided the original work is properly cited.

Networked and cyber-physical system (NCPS) represents a system which tightly integrates computation, communication, and physical processes. Typical NCPS is open, dynamic, and heterogeneous in many dimensions and often needs to be rapidly instantiated and deployed for a given mission. NCPS can be found in areas as diverse as aerospace, automotive, chemical processes, flexible manufacturing systems, medical systems, unmanned vehicles, and traffic control. Despite its high complexity, NCPS has many challenges both in theory and applications. The main focus of this special issue is on the most recent theoretical developments, results, and applications in the area of methods for NCPS.

This special issue collected seven papers that represent a sample of current developments in networked and cyberphysical system. The paper entitled "JAUS to EtherCAT bridge: toward real-time and deterministic joint architecture for unmanned systems" presents a basic joint architecture for unmanned systems (JAUS) implementation in order to evaluate the performance of the standard at its core. By using EtherCAT for internal controls and JAUS as the subsystem level protocol, both interoperability and performance can be achieved and demonstrated in the paper.

The paper with title "Improved weighted Shapley value model for the fourth party logistics supply chain coalition" first analyzes the fourth party logistics supply chain coalition profit allocation models, the classical Shapley value method. Then the weight of individual enterprise in the coalition by the analytic hierarchy process is analyzed. The numerical study shows that the profit allocation method combining Shapley value and distribution according to contribution is relatively rational and practical and is a useful profit allocation mechanism for the fourth party logistics supply chain coalition.

The paper titled "Mobile robot path planning using polyclonal-based artificial immune network" presents polyclonal-based artificial immune network (PC-AIN) for mobile robot path planning. Immunity polyclonal algorithm (IPCA) increases the diversity of antibodies which tend to the same extreme value and finally selects the antibody with the highest concentration. Meanwhile, immunity polyclonal algorithm effectively solves the problem of local minima caused by artificial potential field during the structure of parameter in artificial immune network. Extensive experiments show that the proposed method not only solves immature convergence problem of artificial immune network but also overcomes local minima problem of artificial potential field. So, mobile robot can avoid obstacles, escape traps, and reach the goal with optimum path and faster convergence speed.

The paper titled "Integrity design for networked control systems with actuator failures and data packet dropouts" presents the integrity design problem of fault tolerant control for networked control system (NCS) with actuator failures and data packet dropouts. The data packet dropouts in both sensor-controller (S-C) and controller-actuator (C-A) links are described by two switches, which can be modeled as 
a discrete event system with known rate. After introducing the matrix of actuator failure, the closed-loop NCS is developed, which can be viewed as asynchronous dynamical systems (ADSs). Then, the sufficiency of exponential stability for the NCS is obtained based on the theory of ADSs. The output feedback controllers that can guarantee system stability are also proposed. Finally, two numerical examples are given to demonstrate the validity of our proposed approach.

The paper with title "Research on feature extraction of indicator card data for sucker-rod pump working condition diagnosis" studies three feature extraction methods of suckerrod pump indicator card data, which are Fourier Descriptors (FD), Geometric Moment Vector (GMV), and Gray Level Matrix Statistics (GLMX), respectively. Numerical experiments show that the Fourier Descriptors algorithm requires less running time and less memory space with possible loss of information due to nonoptimal numbers of Fourier Descriptors, the Geometric Moment Vector algorithm is more time consuming and requires more memory space, while the Gray Level Matrix Statistics algorithm provides low-dimensional feature vectors with more time consumed and more memory space. Furthermore, the characteristic of rotational invariance, both in the Fourier Descriptors algorithm and the Geometric Moment Vector algorithm, may result in improper pattern recognition of indicator card data when used for sucker-rod pump working condition diagnosis.

The paper "Real-time detection of application-layer DDoS attack using time series analysis" presents a novel approach to detect application-layer DDoS attack based on entropy of HTTP GET requests per source IP address (HRPI). By approximating the adaptive autoregressive (AAR) model, the HRPI time series is transformed into a multidimensional vector series. Then a trained support vector machine (SVM) classifier is applied to identify the attacks. The experiments with several databases are performed and results show that this approach can detect application-layer DDoS attacks effectively.

The paper "An improved differential evolution algorithm based on adaptive parameter" proposes an adaptive parameter adjustment method which can dynamically adjust control parameters according to the evolution stage. The experiments on high dimensional function optimization showed that the improved algorithm has more powerful global exploration ability and faster convergence speed.

Journal of Control Science and Engineering will continue to attract and disseminate original, theoretically advanced, and practically advantaged papers pertinent to Control Science and Engineering.

Jason $\mathrm{Gu}$

Xiaomei Qi

Ying Wang

Fei Liu

Chengjin Zhang 



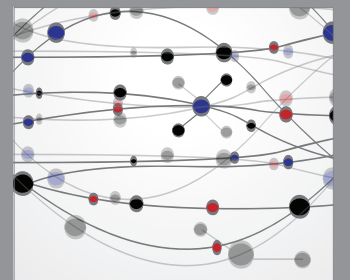

The Scientific World Journal
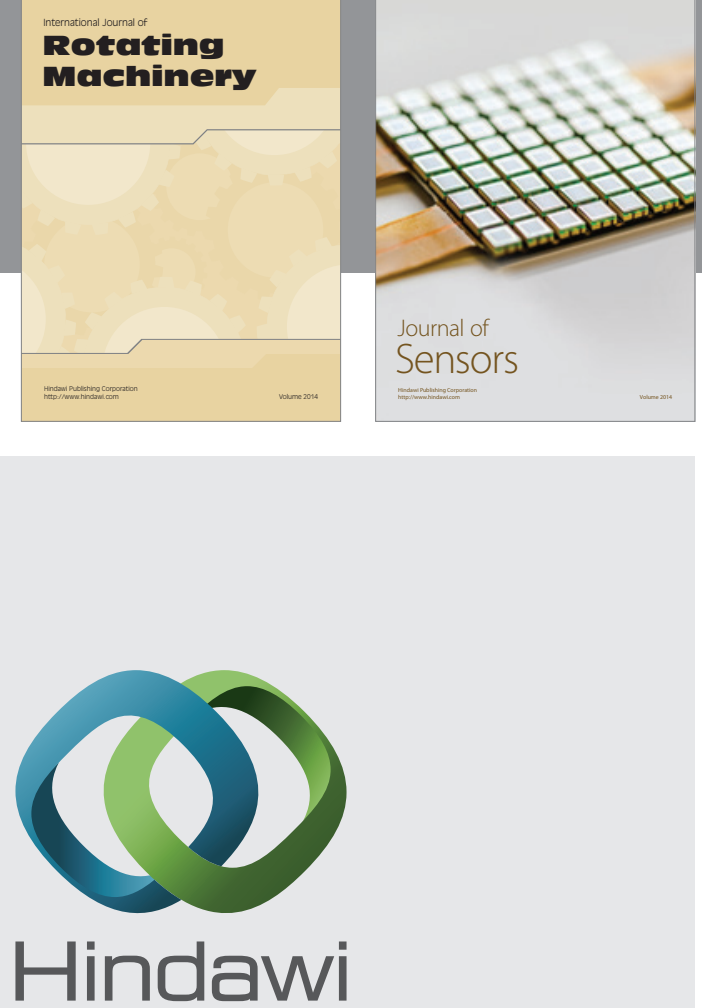

Submit your manuscripts at http://www.hindawi.com
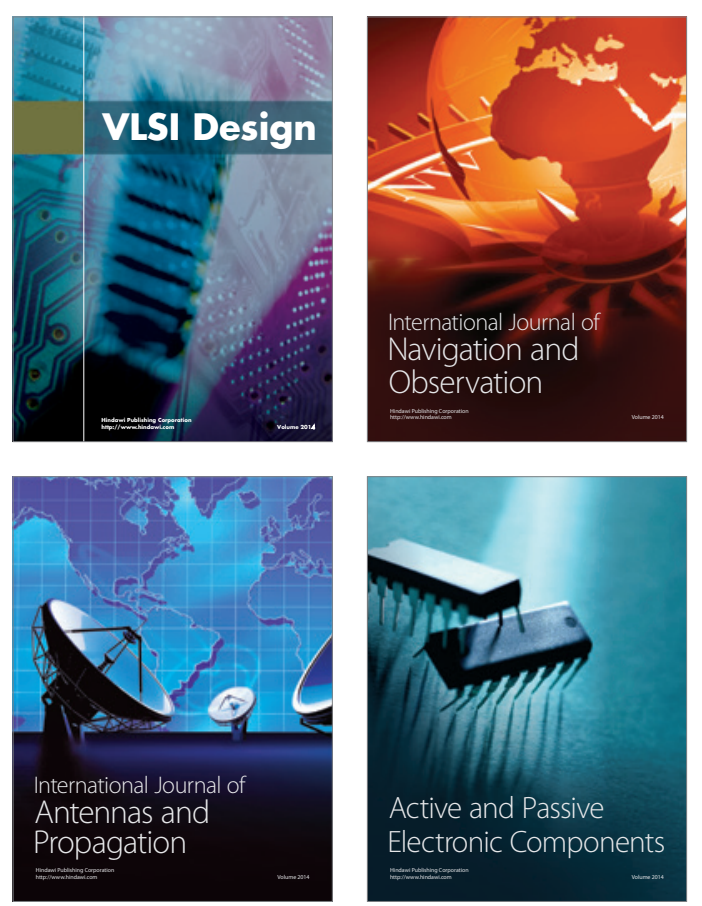
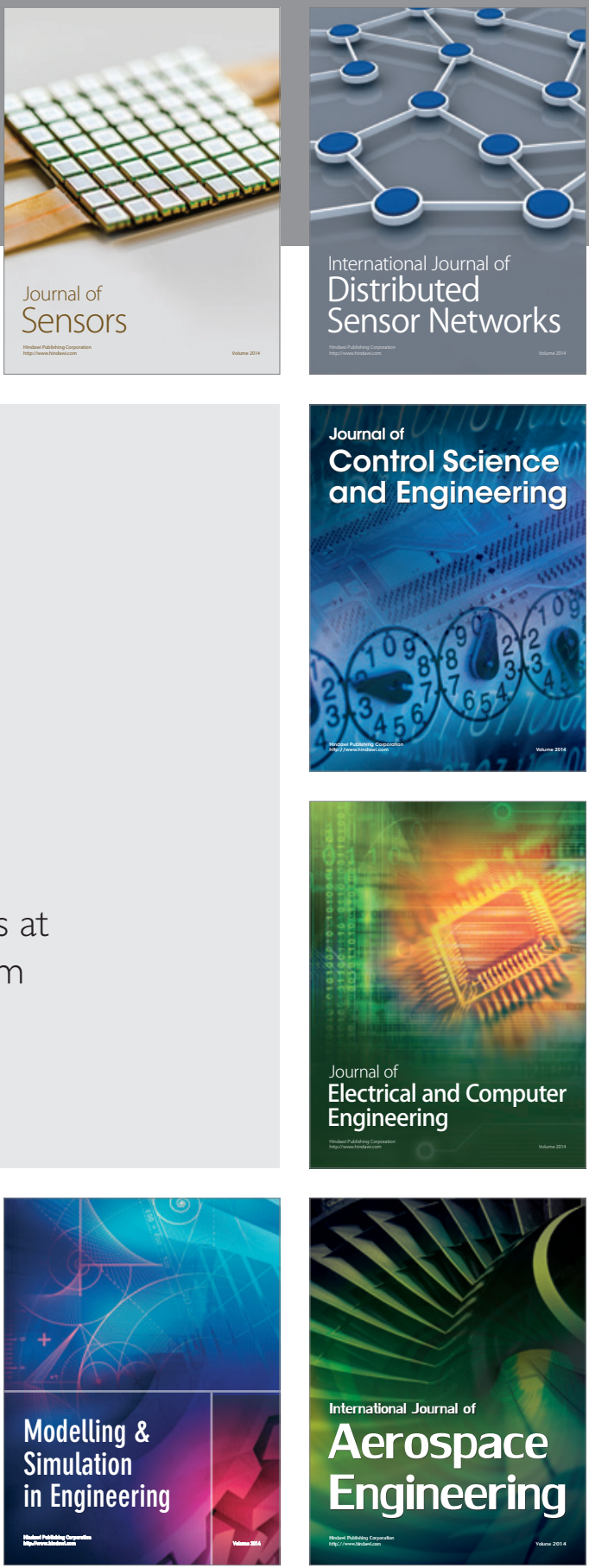

Journal of

Control Science

and Engineering
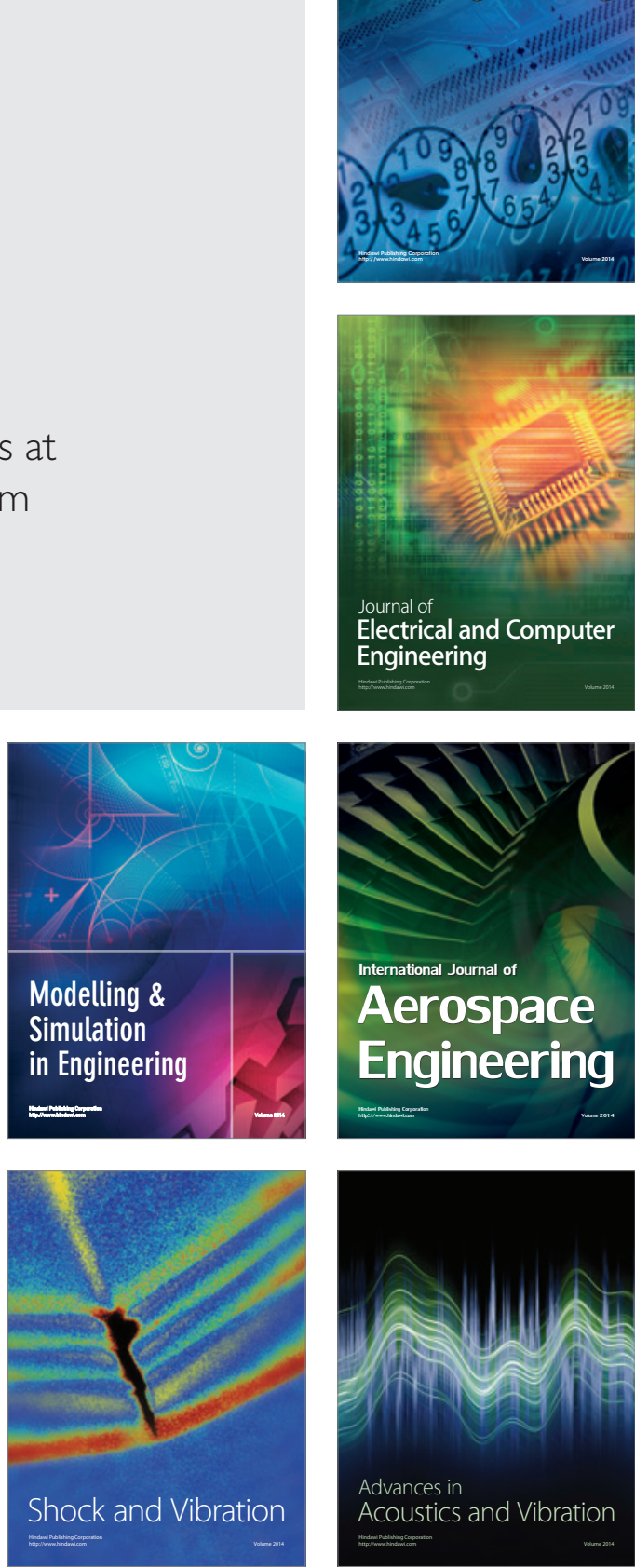